\title{
ESTIMACION DE LA RADIACION SOLAR EN IQUITOS
}

(*) JULIAN BENITES C.

\section{RESUMEN}

Considerando como parámetros la latitud de la ciudad de Iquitos, el ángulo horario, la declinación solar y los registros de hora del sol de la estación meteorológica de Zúngarococha, para el período 1975 a 1985, se reporta por primera vez, valores estimados para los promedios mensuales de radiación solar diaria, que inciden sobre una superficie horizontal en la parte superior de la atmósfera. También se da a conocer los valores estimados para los promedios mensuales de radiación global diaria, que inciden sobre una superficie horizontal para el área de Iquitos. Esta radiación global encontrada es analizada en términos de radiación directa y difusa. Cuando el área de Iquitos cuente con instrumentos que registren la incidencia de radiación solar, los estimados del presente trabajo podrán ser reajustados.

\section{SUM MARY}

Taking as parameters the latitude of Iquitos, the sunset hour angle efor an horizontal surface, the solar declination and sunshine hours (Zungarococha 1975 1985) this paper reports, for the first time, the estimated values of monthly mean daily solar radiation on a horizontal surface for the absence of any atmosphere for the area of Iquitos. It also reports the monthly mean daily global radiation on a horizontal surface for the same area. The global radiation found here is analized in term of direct and diffuse solar radiation. In the future, when Iquitos could count on instruments to measure and record data on solar radiaction; the estimated results from this paper will be improved.

PALABRAS CLAVES: Radiación solar global.

(*) U niversidad Nacional de la A mazonía Peruana Facultad de Ingeniería Química 


\section{INTRODUCCION}

Conocer la incidencia de radiación solar en la región amazónica es de suma importancia, porque este conocimiento permitirá hacer una evaluación de su potencial aprovechamiento, así como de su grado de influencia en los procesos fotosintéticos de diversos recursos naturales de la región. En la actualidad, no se conoce la radiación solar que incide en el área de Iquitos; por eso, el presente trabajo es un esfuerzo inicial orientado a establecer valores estimados de radiación solar que inciden sobre una superficie horizontal.

Considerando que en Iquitos no se cuenta con instrumentos que registren incidencia de radiación solar, se ha recurrido a la utilización de fórmulas que han sido desarrolladas para calcular la radiación solar en una determinada ubicación geográfica, considerando como parámetros la latitud, el ángulo horario, la declinación solar y los registros de horas de sol. En este trabajo, todos estos parámetros están referidos al área de Iquitos. Las horas de sol han sido tomadas de los registros del SENAMHI, correspondientes a la Estación M eteorológica de Zungarococha, para el período 1975 a 1985.

Por falta de disponibilidad de información, en este trabajo no se están considerando otros parámetros que influyen en la incidencia de radiación solar, tales como humedad relativa, temperatura media y precipitación pluvial. Estos parámetros se recomiendan ser tomados en cuenta en futuros trabajos de radiación solar en la región amazónica.

\section{MATERIALES Y METODOS}

Se utilizaron los registros de hora de sol de la estación M eteorológica de Zungarococha, correspondiente a los años 1975 a 1985; asimismo, se calculó (ver 1 y 2) la declinación y el ángulo horario para los 365 días del año, correspondientes a la latitud de Iquitos. Con esta información se recurrió al uso de relaciones matemáticas empíricas para estimar la radiación solar que incide en Iquitos. EF análisis se centro en cuatro aspectos fundamentales insolación, radiación, que incide en la parte superior de la atmósfera, radiación global, que incide a nivel del suelo en el área de Iquitos y el análisis de la radiación global, en términos de radiación directa y radiación difusa. 


\section{RESULTADOS Y ANALISIS}

\section{Insolación}

Utilizando los registros de horas de sol de Zungarococha, se determinó la insolación real ñ o promedio mensual del número diario de horas de sol registradas en la estación meteorológica. El valor encontrado por mes es durante el período 1975 1985 se muestra en la Tabla 1. En esta tabla se puede apreciar que ñ toma un valor máximo de 5.16 en el mes de agosto y un valor mínimo de 3.25 en el mes de abril. En la misma Tabla, se muestra que la insolación real en invierno es de 4.78 horas de sol diarias, mientras que en el verano es de solamente 3.77 horas de sol diarias. También se puede apreciar que el número anual de sol para el período 1975 1985, ha variado entre 1206 y 1724 horas de sol por año, siendo la mas al ta la correspondiente a 1979 y la mas baja a 1984 .

El curso anual del promedio mensual del número diario de horas de sol ñ, así como su variación con respecto a las estaciones del año, se muestran en la Figura 1.

FIGURA 1

\section{CURSO DEL PROMEDIO MENSUAL DEL NUMERO DIARIO DE HORAS} DE SOL O INSOLACION REAL ñ.

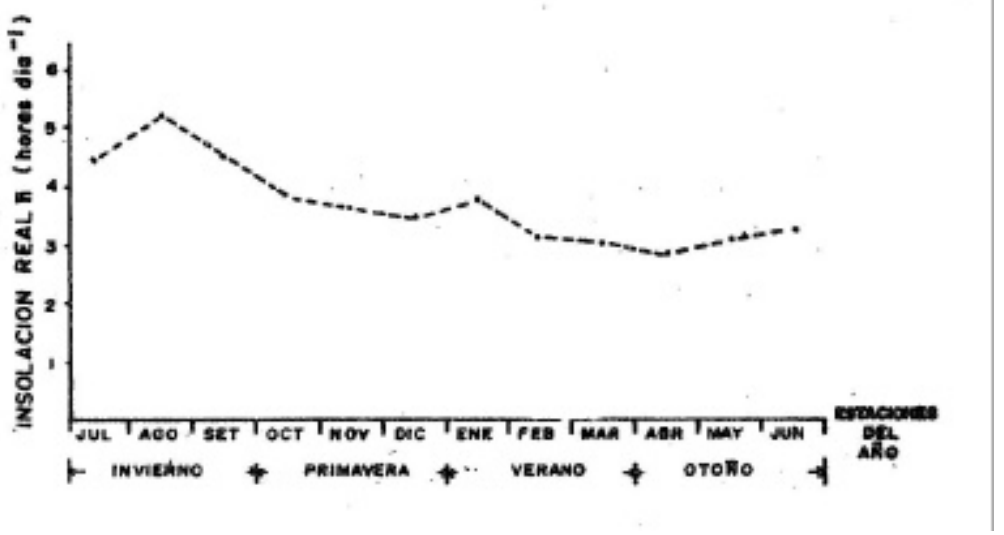


TABLA 1.

NUMERO MENSUAL DE HORAS DE SOL PARA LA ESTACION DE ZUNGAROCHOCA EN IQUITOS, ENTRE 1975 - 1985

\begin{tabular}{clllllllllllll}
\hline Año & Enero & Feb. & Mar. & Abr. & May. & Jun. & Jul. & Ago & Set. & Oct. & Nov. & Dic. Total de Horas de \\
1975 & 120.3 & 88.6 & 130.5 & 122.0 & 92.6 & 82.6 & 137.8 & 167.6 & 144.6 & 134.8 & 134.5 & 147.2 & sol Anual \\
1976 & 116.2 & 113.0 & 92.2 & 86.6 & 108.0 & 139.5 & 147.4 & 180.0 & 150.3 & 130.5 & 112.9 & 122.3 & 1498.10 \\
1977 & 145.1 & 101.8 & 94.8 & 104.3 & 116.4 & 121.1 & 183.9 & 169.1 & 108.1 & 117.9 & 111.9 & 136.1 & 1498.90 \\
1978 & 141.9 & 119.2 & 115.2 & 97.8 & 121.7 & 127.6 & 127.3 & 159.5 & 144.1 & 156.1 & 139.9 & 117.8 & 1510.50 \\
1979 & 167.9 & 95.2 & 138.2 & 105.2 & 136.1 & 127.6 & 165.2 & 174.7 & 171.7 & 167.1 & 127.1 & 148.2 & 1568.10 \\
1980 & 167.9 & 93.6 & 73.7 & 106.0 & 135.8 & 110.2 & 139.1 & 191.5 & 164.3 & 119.0 & 123.1 & 162.0 & 1724.20 \\
1981 & 136.2 & 78.0 & 114.5 & 106.7 & 123.2 & 110.8 & 148.2 & 154.0 & 140.3 & 159.8 & 131.5 & 117.8 & 1586.20 \\
1982 & 104.0 & 88.5 & 101.5 & 76.0 & 78.0 & 147.2 & 113.5 & 133.0 & 96.7 & 126.0 & 104.5 & 97.0 & 1521.00 \\
1983 & 123.5 & 106.7 & 99.7 & 89.6 & 109.0 & 93.3 & 142.5 & 114.0 & 159.5 & 96.0 & 129.0 & 80.5 & 1265.90 \\
1984 & 127.5 & 83.0 & 86.7 & 73.0 & 89.5 & 69.5 & 103.5 & 104.0 & 110.0 & 109.5 & 127.5 & 122.5 & 1347.00 \\
1985 & 159.5 & 100.6 & 116.8 & 104.0 & 103.0 & 117.5 & & & & & & & 1206.20 \\
\hline
\end{tabular}

Promedio

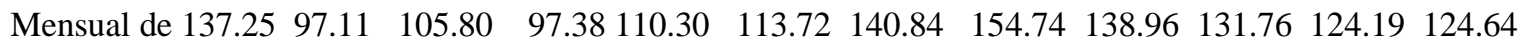

Horas de sol

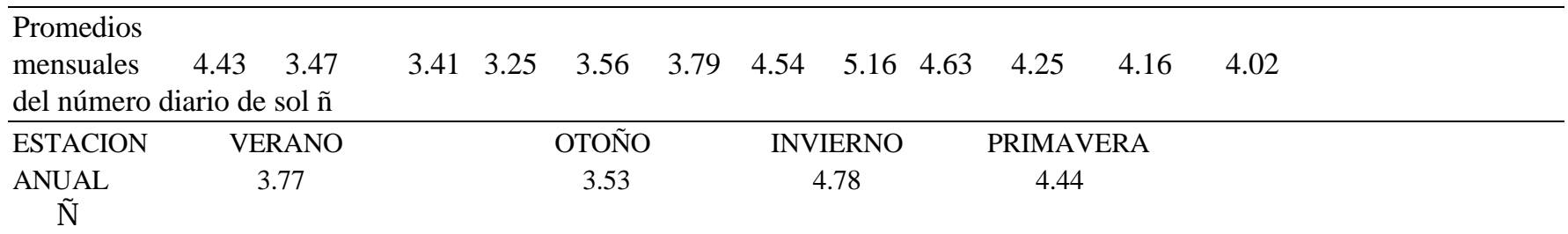


Peter Teagan (1) ha reportado para estaciones de selva alta, como Chachapoyas, Iberia, San Ramón y San Jorge. un promedio mensual del número diario de horas de sol de 5.78, es decir, un promedio aproximadamente de dos horas de sol diarias mayores que las registradas en Zungarococha.

Utilizando la latitud de Iquitos $L=03^{\circ} 45^{\prime} S$ y la formula para calcular la de eliminación solar :

$$
?=2345^{\circ} \operatorname{Sen}\left\{\frac{360(284+D)}{365}\right\} \operatorname{con} 1=\mathrm{D}=365 \text {, propuesta por } \mathrm{J} \text {. }
$$

A. Duffiee y W. A. Beckman (2), se calculó la insolación teórica para cada día del año correspondiente a la latitud de Iquitos, a través de la relación reportada por $M$. K. El . A dawi et. al. (3).

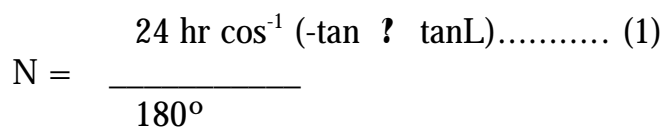

Esta expresión proporciona el número teórico o número posible de horas de brillo solar que, teóricamente, deberían observarse en Iquitos para cada día del año. U tilizando la ecuación (1) se encontró el número teórico de horas de brillo solar de los 365 días para la latitud de Iquitos, estos valores encontrados se agruparon para cada mes del año y se encontró un valor $\tilde{\mathbf{N}}$ para cada mes, que está representando el promedio mensual del número diario de horas de sol teóricas que se espectarían ser observadas en Iquitos. En otras palabras, Ñ es el promedio mensual del número diario de horas de sol teórico o insolación teórica. A nalizando los valores encontrados para $\tilde{\mathbf{N}}$ se observa que, a partir de abril hasta setiembre, $\tilde{\mathbf{N}}$ es menor que 12 horas diarias, pero, a partir de octubre hasta marzo, $\tilde{\mathbf{N}}$ es mayor que 12 horas diarias. La variación máxima para $\tilde{\mathbf{N}}$ correspondiente a la latitud de Iquitos, es de aproximadamente 0,5 horas día $a^{-1}$ y corresponde a la diferencia de $\tilde{\mathbf{N}}$ correspondiente a diciembre y junio. Esta información está consolidada en la Tabla 2 y representada en la Figura 2, que se presentan a continuación. 
TABLA 2

\section{PROMEDIOS MENSUALES DE LA INSOLACION TEORICA ÑPARA EL AREA DE IQUITOS}

\begin{tabular}{|lllllllllll|}
\hline $\begin{array}{l}\text { M eses Julio A gosto } \\
\text { Jun. }\end{array}$ & Set. & Oct. & Nov. & Dic. & Ene. & Feb. & M arz & Abr. & M ay. \\
\hline $\begin{array}{l}\tilde{\mathbf{N}} \mathbf{1 1 . 8 0} \\
11.78\end{array}$ & 11.88 & 11.98 & 12.08 & 12.17 & 12.20 & 12.17 & 12.12 & 12.02 & 11.91 & 11.83 \\
\hline
\end{tabular}

\section{FIGURA 2}

\section{CURSO ANUAL DE LA INSOLACION TEORICA Ñ PARA LA LATITUD 33은 45' S CORRESPONDIENTE A IQUITOS.}

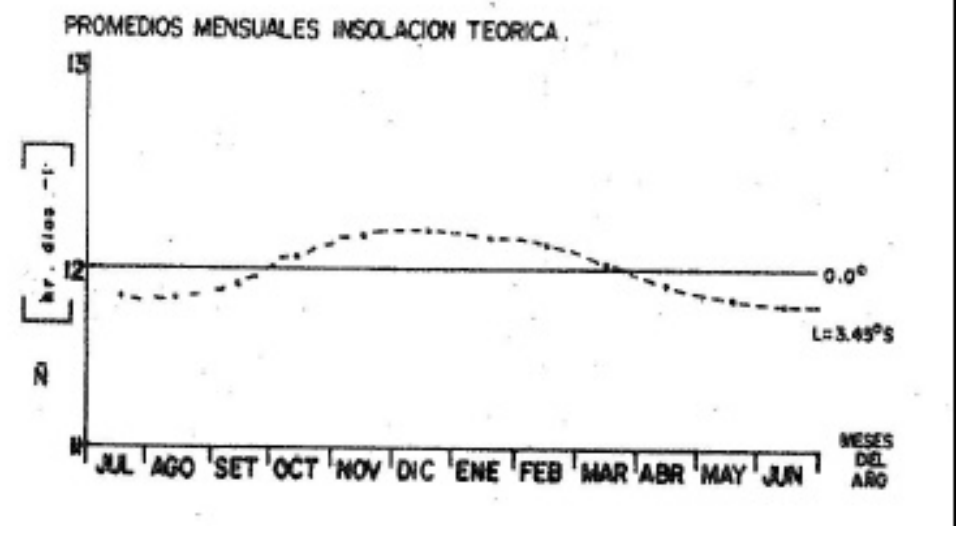

Habiéndose obtenido los valores de insolación real u (promedio mensual del número diario de horas de sol registradas en la Estación Zúngarococha) y de la insolación teórica $\tilde{\mathbf{N}}$ (promedio mensual del número diario de horas de sol teóricas espectadas para el área de lquitos), es posible establecer la insolación relativa o razón de insolación, como la razón de:

$$
\frac{\tilde{n}}{\tilde{N}}
$$

Este factor de la insolación relativa, va a ser utilizada en la sección final de este trabajo para calcular la radiación solar global que incida en el área de Iquitos. En la Tabla 3 y la Figura 3, que se presentan a continuación, se puede observar que la considerable diferencia entre los valores de $\tilde{n}$ y $\tilde{N}$, implican valores pequeños para 
que, en definitiva, van a influenciar en los cálculos de radiación que incidan en el área de Iquitos. Por otro lado, considerando que los valores para son casi uniformes, todas las tendencias de la variación anual de 1 en Iquitos, estarán fuertemente influenciadas básicamente por las variaciones de ñ.

\section{TABLA 3}

\section{RAZON DE LA INSOLACION PARA EL AREA DE IQUITOS, CORRESPONDIENTE A LOS AÑOS 1975 - 1985}

Meses Ene. Feb. Mar. Abr. May. Jun. Jul. Ago. Set. Oct. Nov. Dic.

$\begin{array}{cccccccccccccc} & 4.43 & 3.47 & 3.41 & 3.25 & 3.56 & 3.79 & 4.54 & 5.16 & 4.63 & 4.25 & 4.16 & 4.02 \\ \frac{\tilde{n}}{\tilde{N}} & 12.17 & 12.12 & 12.02 & 11.91 & 11.83 & 11.78 & 11.80 & 11.88 & 11.98 & 11.08 & 12.17 & 12.20 \\ \frac{\tilde{n}}{\tilde{N}} & 0.36 & 0.29 & 0.28 & 0.27 & 0.30 & 0.32 & 0.38 & 0.43 & 0.39 & 0.35 & 0.34 & 0.33\end{array}$

FIGURA 3

VARIACION ANNUAL DE LA RAZON DE INSOLACION O INSOLACION RELATIVA PARA IQUITOS

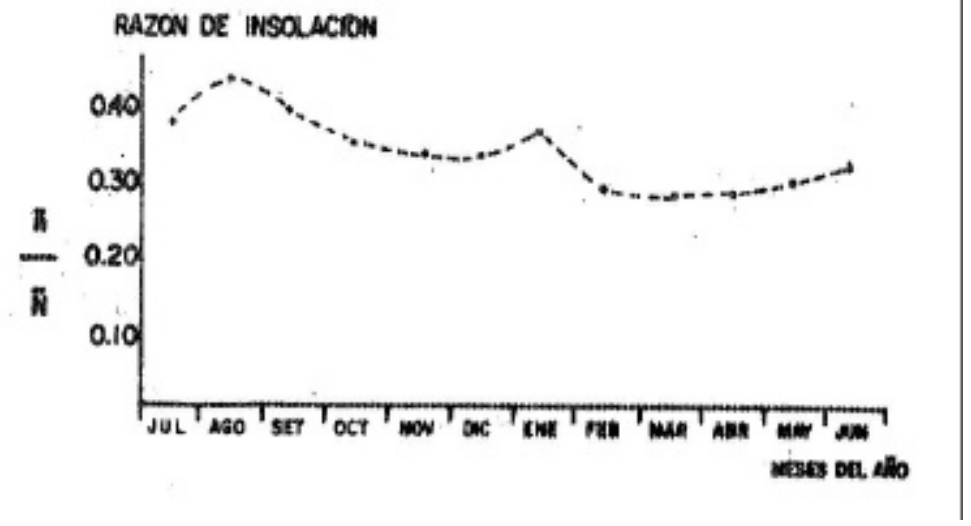

Radiación solar diaria que incide sobre una superficie horizontal en la parte superior de la atmósfera correspondiente a la latitud de Iquitos $\mathrm{H}_{0}$. 
Para calcular la radiación solar diaria que incide sobre un plano horizontal en la parte superior de la atmósfera, se realizó la ecuación propuesta por S.A. K lein (5) y utilizada par Said. M. A. Ibrahim (6), que es la siguiente:

$$
\begin{aligned}
& H_{O}=\frac{24 h r . d i a^{-1} I}{\pi} \operatorname{sc}\left[1+0.033 \operatorname{Cos} \frac{(360 D}{365}\right] \\
& x\left[\operatorname{Cos} L \operatorname{Cos} \int \operatorname{sen} \omega+\frac{(2 \pi \omega)}{360} \operatorname{senLSen} \int\right] \ldots((2))
\end{aligned}
$$

En esta fórmula, I = $4871 \frac{\mathrm{kj}}{\mathrm{hrm}^{2}} \quad$ I es la constante solar; $1=\mathrm{D}=365$ es cada día del año; $L=030$ 45'S es la latitud de Iquitos; ? son los valores diarios de la declinación solar, calculados en la sección anterior por la fórmula propuesta en (2); por último ? $=\cos ^{-1}(-\tan \mathrm{L} \tan ?)$, es el ángulo horario, de acuerdo a la fórmula propuesta por J. P. Chiou y M M. EI Naggar (4).

Se calcularon los valores del ángulo horario correspondiente a los 365 días del año y estos valores fueron utilizados para calcular los correspondientes valores diarios de $\mathrm{H}_{0}$ en la fórmula (2). Los valores diarios de $\mathrm{H}_{0}$ para los 3.65 días del año se agruparon por meses y se calculé un promedio mensual de radiación solar diaria, que incide sobre una superficie horizontal en la parte superior de la atmósfera, la cual se denominará Ho. Los valores correspondientes a $\mathrm{H}_{0}$ aparecen en la Tabla 4 y el curso anual correspondiente en la Figura 4.

\section{TABLA 4.}

\section{PROMEDIOS MENSUALES DE LA RADIACION SOLAR DIARIA Ho QUE INCIDE EN LA PARTE SUPERIOR DE LA ATMOSFERA SOBRE UNA SUPERFICIE HORIZONTAL CORRESPONDIENTE A AL LATITUD DE IQUITOS}

\begin{tabular}{|l|l|l|l|l|l|l|l|l|l|l|l|l|}
\hline M eses & Jul. & A go. & Set. & Oct. & Nov. & Dic. & Ene. & Feb. & M ar. & A br. & M ay. & Jun. \\
\hline $\begin{array}{l}\mathrm{Ho}\left(\mathrm{M} \mathrm{n}^{-}\right. \\
\left.{ }^{2} \mathrm{dia}^{-1}\right)\end{array}$ & 33.55 & 35.23 & 36.90 & 37.10 & 36.23 & 35.89 & 35.98 & 36.90 & 37.21 & 35.96 & 34.04 & 33.15 \\
\hline
\end{tabular}




\section{FIGURA 4}

CURSO ANUAL DE LOS PROMEDIOS MENSUALES DE LA RADIACION SOLAR DIARIA Ho QUE INCIDE EN LA PARTE SUPERIOR DE LA ATMOSFERA SOBRE UNA SUPERFICIE HORIZONTAL PARA LA LATITUD DE IQUITOS L = 03 45' S; COMPARADOS CON LOS VALORES ENCONTRADOS POR N.A. VILLA NOVA et. AI. PARA LA LATITUD DE MANAOS $\varnothing=03$ 0 08'S

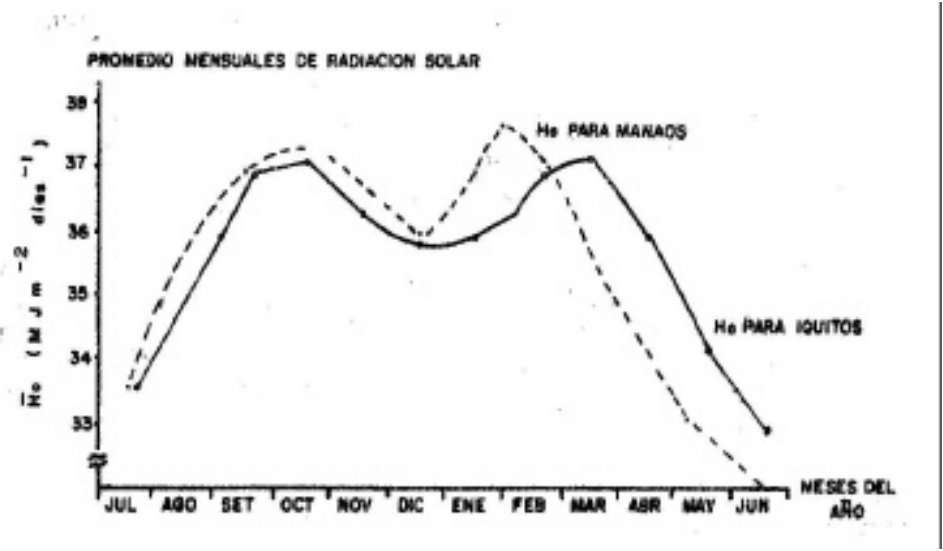

En la figura 4, se puede apreciar que la variación anual de $\mathrm{H}_{0}$ calculada para el área de Iquitos, con latitud $\mathrm{L}=03^{\circ} 45^{\prime} \mathrm{S}$, sigue la tendencia de los valores reportados por N. A. Villa Nova et. al. (7), para la latitud de Manaos $\varnothing=030$ 08' S. Sin embargo, es preciso indicar que esta comparación es solamente referencial, por dos razones: primero, porque las latitudes son solamente aproximadas y, segundo, que V illa N ova ha utilizado la fórmula:

$$
C O=\frac{1440 X I o}{\pi R^{2}}(\operatorname{Cos} \phi \operatorname{Cos} 6 \operatorname{Senh} p-h p \operatorname{Sen} \phi \operatorname{Sen} 6)
$$

Sen ( Sen, propuesta por J. C. Ometto (8), que es menos completa que la fórmula (2) propuesta por 5. A. Klein y utilizada en el presente trabajo para calcular $\mathrm{HO}$ en Iquitos.

Promedio M ensual de Radiación Solar Global Diaria que Incide en una Superficie Horizontal para el área de Iquitos $\mathrm{H}$. 
El modelo utilizado en el presente trabajo para calcular el promedio mensual de radiación solar global diaria que incide en una superficie horizontal en el área de Iquitos, es el propuesto por A. A ngstrom (9), que tiene la fórmula siguiente:

$$
\frac{H}{H_{O}}=a+b\left(\frac{\tilde{n}}{\tilde{n}}\right) \ldots \ldots .(3)
$$

Las constantes $a$ y $b$ de este modelo de regresión lineal propuestos por A ngstrom, pueden ser calculados por el método de los mínimos cuadrados, siempre que se dispongan de los promedios mensuales de radiación global diaria II, registrados en el sitio donde se va aplicar la fórmula.

Para el caso de Iquitos, basta la fecha no me cuenta con registros de promedios mensuales de radiación solar global diaria; esta realidad no permite encontrar las constantes a y b con la ecuación (3) y esto, a su vez, imposibilita por ahora determinar una ecuación exacta para determinar los promedios mensuales de radiación solar global diaria para el área de Iquitos.

Sin embargo, existe una aproximación propuesta por J. Glover and J.S.F. M c. Culloch (10), que permite estimar los valores de a y b de la ecuación (3) en los lugares donde no existen registros para $\mathrm{H}$, como es el caso de Iquitos. A nte esta situación se optó por la siguiente aproximación:

Para el valor de a se utilizó la aproximación propuesta por Glover y M c Culloch en (10):

$$
a=0.29 \operatorname{Cos} L ; \operatorname{con} L=03^{\circ} 45^{\prime} S
$$

Para el valor de b se decidió utilizar un promedio de valor propuesto por Glover y Mc Culloch, que es igual a 0.52 , y el propuesto por L. Oldeman and M. Frere (11), que es igual a 0.42 , en razón de que ambos trabajos están referidos al trópico, especialmente el último, que se ha realizado para trópicos húmedos. siguiente:

Con estas aproximaciones para a y b, la ecuación (3) toma la forma final

$$
\frac{H}{\mathrm{H}_{\mathrm{O}}}=0.29+0.47\left(\frac{\tilde{n}}{\tilde{N}}\right) \cdots \cdots \cdots
$$


En base de esta ecuación (4), conjuntamente con los resultados de la Tabla 3 para y los de la tabla 4 para los valores $\mathrm{Ho}$, se ha estimado los promedios mensuales de radiación solar global diaria para Iquitos $\mathrm{H}$. Los resultados de los cálculos para $\mathrm{H}$ se muestran en la Tabla 5. A nalizando la Tabla 5, se puede apreciar que los valores de la radiación solar que no llegan a alcanzar la superficie de la tierra y que se pierden en proceso de absorción, extinción y dispersión, es del orden del 54\%.K.G. Barry y S.K. Chorley (12), reportan una perdida de radiación del $57 \%$ para estos mismo procesos, que es un valor próximo al encontrado en este trabajo de aproximación.

Si comparamos el curso anual de $\mathrm{H}$ Ode la Figura 4 con el curso anual de $\mathrm{H}$ de la Figura 5, se puede observar lo siguiente: Ho muestra dos máximos más o menos iguales correspondiente a los meses de setiembre y marzo, mientras que $\mathrm{H}$ muestra un sólo máximo correspondiente al mes de setiembre, mas no se aprecia el máximo correspondiente al mes de marzo.

Esto estaría indicando que el máximo de radiación solar Ho que llega a la parte superior de la atmósfera, estaría siendo influenciado por factores atmosféricos y climatológicos propios del área de Iquitos, para esta época del año. 
128

\section{TABLA 5}

PROMEDIOS MENSUALES DE RADIACION GLOBAL DIARIA QUE INCIDE SOBRE UNA SUPERFICIE HORIZONTAL EN EL AREA DE IQUITOS H

\begin{tabular}{|c|c|c|c|c|c|c|}
\hline \multirow[b]{2}{*}{$M$ eses } & & \multicolumn{3}{|c|}{$\mathrm{HO}$} & \multicolumn{2}{|r|}{$\mathrm{H}$} \\
\hline & $\tilde{n}$ & $\left(\mathrm{M} J \mathrm{~m}^{-2}\right.$ días $\left.^{-1}\right)$ & $a$ & $b$ & $\left(\mathrm{M} J \mathrm{~m}^{-2}\right.$ días $\left.^{-1}\right)$ & \% de radiación \\
\hline & $\tilde{\mathbf{N}}$ & & & & & a la tierra \\
\hline
\end{tabular}

$\begin{array}{lllllll}\text { Ener. } & 0.36 & 35.98 & 0.29 & 0.47 & 16.52 & 54 \% \\ \text { Feb. } & 0.29 & 36.90 & 0.29 & 0.47 & 15.73 & 57 \% \\ \text { M ar. } & 0.28 & 37.21 & 0.29 & 0.47 & 15.69 & 57 \% \\ \text { A br. } & 0.27 & 35.96 & 0.29 & 0.47 & 14.99 & 58 \% \\ \text { M ay. } & 0.30 & 34.04 & 0.29 & 0.47 & 14.67 & 57 \% \\ \text { Jun. } & 0.32 & 33.15 & 0.29 & 0.47 & 14.60 & 56 \% \\ \text { Jul. } & 0.38 & 35.55 & 0.29 & 0.47 & 16.66 & 53 \% \\ \text { A gos. } & 0.42 & 35.23 & 0.29 & 0.47 & 17.17 & 51 \% \\ \text { Set } & 0.39 & 36.90 & 0.29 & 0.47 & 17.46 & 52 \% \\ \text { Oct. } & 0.35 & 37.10 & 0.29 & 0.47 & 16.86 & 54 \% \\ \text { N ov. } & 0.34 & 36.23 & 0.21 & 0.47 & 15.30 & 55 \% \\ \text { Dic. } & 0.33 & 35.89 & 0.29 & 0.47 & 15.97 & 55 \%\end{array}$




\section{FIGURA 5}

\section{CURSO ANNUAL DE LOS PROMEDIOS MENSUALES DE LA RADIACION GLOBAL DIARIA QUE INCIDE SOBRE UNA SUPERFICIE HORIZONTAL EN EL AREA DE IQUITOS Ho}

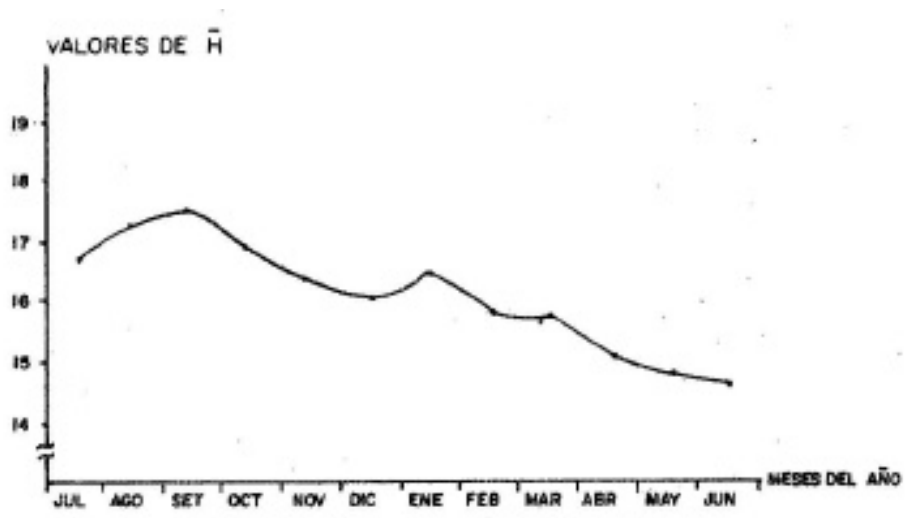

\section{ANALISIS DE LA RADIACION GLOBAL ENCONTRADA EN TERMINOS DE RADIACION DIRECTA Y RADIACION DIFUSA.}

Los promedios mensuales de radiación solar global diaria II que se ha encontrado para el área de Iquitos, está compuesta de radiación directa y radiación difusa, de acuerdo a la siguiente relación:

$H=H_{B}+H_{D}$, donde $H_{B}$ es la radiación directa y $H_{D}$ es la radiación difusa. No existiendo para Iquitos registros de radiación difusa $\mathrm{H}_{\mathrm{D}}$, se ha efectuado un estimado de radiación difusa para el área de Iquitos, utilizando una correlación entre radiación global H y radiación difusa Ho, propuestos por B.Y.H. Liu and R.C. Jordan (13), que es la siguiente:

$\frac{H_{D}}{H}=1.0-1.112 \mathrm{Kr}$

A quí, HD es el promedio mensual de la radiación solar difusa diaria que incide sobre una superficie horizontal y $\mathrm{Kr}=\frac{\mathrm{H}}{\mathrm{H}_{\mathrm{O}}}$, es el índice de la claridad, 
En la Tabla 6 se presentan los valores calculados para $\mathrm{K}_{\mathrm{T}}, \mathrm{HD}_{\mathrm{D}} \frac{\mathrm{H}}{\mathrm{H}_{\mathrm{o}}}$ y HB, utilizando la ecuación (5)

TABLA 6

VALORES PROMEDIOS MENSUALES DE RADIACION SOLAR DIARIA PARA RADIACION SOBRE UNA SUPERFICIE HORIZONTAL EN LA PARTE EXTERIOR DE LA. ATMOSFERA HO. RADIACION SOLAR GLOBAL SOBRE UNA SUPERFICIE HORIZONTAL A NIVEL DEL SUELO H. FACTOR DE LA CLARIDAD K $\mathrm{K}^{=} \frac{H}{H_{0}}$ RADIACION DIRECTA HB. .RADIACION DIFUSA HD.

(MJ $\mathrm{m}^{-2}$ día $^{-1}$ )

MESES Ho $\mathrm{H} \quad \mathrm{H}_{\mathrm{T}}=\frac{H}{H_{o}} \quad \mathrm{H}_{\mathrm{O}}=\mathrm{H}\left(10-1.112 \mathrm{~K}_{\mathrm{T}}\right) \frac{H}{H_{o}} \quad \mathrm{H}_{\mathrm{B}}=\mathrm{H}-\mathrm{H}_{\mathrm{D}}$

\begin{tabular}{lllllll} 
Ene & 35.98 & 16.52 & 0.46 & 8.07 & 0.49 & 8.45 \\
F eb & 36.90 & 15.73 & 0.43 & 8.21 & 0.52 & 7.52 \\
M arz & 37.21 & 15.69 & 0.42 & 8.36 & 0.53 & 7.33 \\
A br & 35.96 & 14.99 & 0.44 & 8.17 & 0.51 & 7.82 \\
M ay & 34.04 & 14167 & 0.43 & 7.66 & 0.52 & 7.01 \\
Jun & 33.15 & 14.60 & 0.44 & 7.46 & 0.51 & 7.14 \\
Jul & 35.55 & 16.66 & 0.47 & 7.95 & 0.48 & 8.71 \\
A go & 35.23 & 17.17 & 0.49 & 7.81 & 0.45 & 9.36 \\
Set & 36.90 & 17.46 & 0.47 & 8.33 & 0.48 & 9.13 \\
Oct & 37.10 & 16.86 & 0.45 & 8.42 & 0.50 & 8.44 \\
N ov & 36.23 & 16.30 & 0.45 & 8.14 & 0.50 & 8.16 \\
Dic & 35.89 & 15.97 & 0.44 & 8.16 & 0.51 & 7.81 \\
\hline
\end{tabular}

Considerando que los valores promedios anuales obtenidos de la Tabla 6 son $H_{0}=35.85 \mathrm{MJ} \mathrm{m}^{-2}$ día $^{-1}$ es el $100 \%$ de radiación que llega a la parte superior de la atmósfera, y que $\mathrm{H}=16,14 \mathrm{MJ} \mathrm{m}^{-2}$ día $^{-1}$ es la radiación global que llega a la superficie del suelo. Es decir, el valor de la radiación global $\mathrm{H}$ que llega al suelo está representado a un $45.02 \%$ de la radiación solar que llegó a la parte superior de la atmósfera. Los porcentajes de radiación que se pierden en la atmósfera por procesos de absorción, extinción y dispersión han sido calculados en la Tabla 5 y representan 
un 54.98\%. Es decir, que la radiación global que llega a la tierra, más la radiación que se pierde en las interacciones con la atmósfera, es igual a la radiación que llega a la parte superior de la atmósfera.

La Radiación difusa $\mathrm{H}_{\mathrm{D}}=8.06 \mathrm{MJ} \mathrm{m}^{-2}$ día $^{-1}$, es el $22.48 \%$ de $\mathrm{H}_{0}$, y la Radiación directa $\mathrm{H}_{\mathrm{B}}=8.05 \mathrm{MJ} \mathrm{m}^{-2}$ día ${ }^{-1}$ es el $22.45 \%$ de $\mathrm{H}_{\mathrm{o}}$. Entonces, de acuerdo a los valores encontrados con la correlación de Liu and Jordan ecuación (5), la radiación global, que es la suma de la radiación difusa más la radiación directa, estaría representando el $44.93 \%$ de la radiación que llega a la parte superior de la atmósfera. Lo que estaría significando un error de $0,2 \%$ con respecto al valor de $45.02 \%$, discutido en el párrafo anterior para la radiación global.

Finalmente, graficando los valores de $H_{D}$ y de $K_{T}$ que aparecen en la Tabla 6, se puede tener una recta que represente la relación de dependencia de la radiación difusa con el índice de claridad. Esa relación de dependencia se presenta en la Figura 6.

\section{FIGURA 6}

DEPENDENCIA LINEAL DE LA RADIACION DIFUSA CON INDICE DE CLARIDAD.

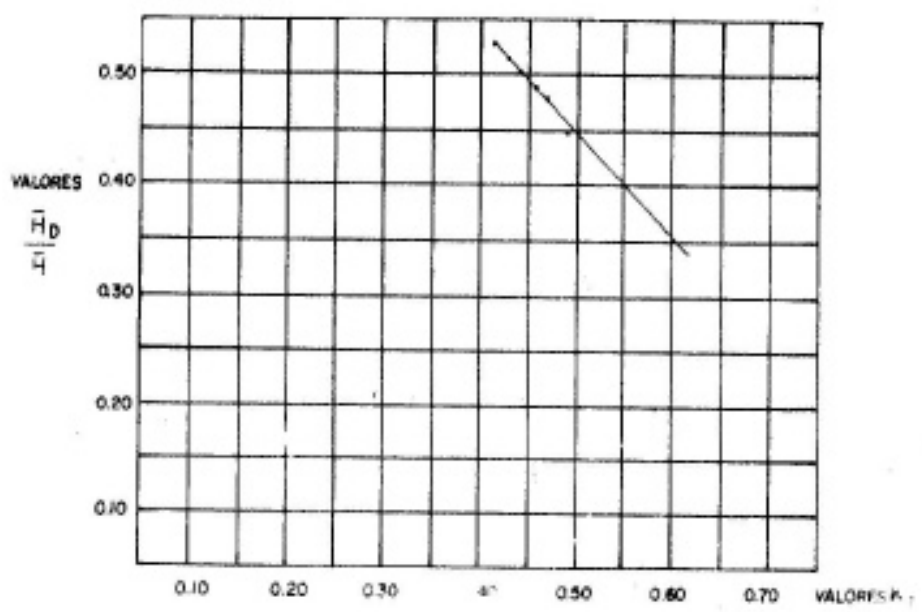


En esta Figura 6 se puede apreciar que, cuanto más pequeño son los índices de claridad, mayores índices de Radiación difusa inciden sobre el área de Iquitos.

Los datos que se han obtenido en el presente trabajo para $\mathrm{H}_{\mathrm{D}}$ son solamente estimaciones hechas con la correlación de Liu and Jordan, por no disponer a la fecha de un registro de datos para $\mathrm{H}_{\mathrm{D}}$ en Iquitos.

Cuando en el futuro se cuenten con estos datos en esta área, se podrá encontrar una ecuación de correlación entre $H_{D}$ y $K_{T}$ de tipo de ecuación (5), con constantes propias para el área de Iquitos; es decir, se podrá establecer una ecuación de correlación para la Radiación Difusa en el área de Iquitos. Al contar con esta información en el futuro se podrán hacer ajustes y correlaciones que requieren los estimados para $H_{D}$ encontrados en el presente trabajo.

\section{CONCLUSIONES}

U tilizando los datos de insolación real de la Estación Zungarococha, se han obtenido valores estimados para los promedios mensuales de radiación global, difusa y directa que inciden en el área de Iquitos y que aparecen en la Tabla 6.

Los resultados para la insolación real, la radiación extraterrestre y la radiación global, siguieron las tendencias de resultados reportados para M anaus. Sin embargo, $M$ anaus reporta un promedio de 2000 horas anuales de insolación real, que es mayor a las 1500 horas de insolación real reportadas para Iquitos en el presente trabajo. Esta diferencia de insolación real estaría significando una diferencia de radiación solar entre estas dos áreas. Sin embargo, para que esta situación sea concluyente, se requiere de futuros estudios orientados a analizar la tendencia de estos resultados.

En futuro, cuando se cuenta con registros para la radiación global, radiación difusa y radiación directa, siguiendo el mismo procedimiento del presente trabajo, se podrán obtener las fórmulas de correlación para calcular con más exactitud las diferentes formas de radiación en el área de Iquitos. Esto permitirá, a su vez, hacer los reajustes necesarios a los valores estimados en el presente trabajo.

En este trabajo inicial sobre el tema de radiación solar en esta área de la amazonía peruana, solamente se han considerado los parámetros de horas de sol, declinación solar y latitud para Iquitos; en el futuro se podrán realizar trabajos sobre este mismo tema y para esta misma área que incluyen otros parámetros, como 
humedad relativa, temperatura media y máxima precipitación pluvial, turbidad, albedo de la tierra.

\section{REFERENCIAS BIBLIOGRAFICAS}

PETER TEAGAN, Potencial use of solar/wind Energy Resources in Peru U.S.A. Peru Cooperative Energy A ssenment. A rthur D. Little, INC. (1978)

J. A. DUfFIEE and W. A. BECKMAN, Solar Energy Thermal Processes Wiley Interscience, N ew Y ork (1974)

M. K. EI - ADAWI, M. M. EI - NIKLAWI, A. A. KUTUB and G.G. AI BARAKATI. Estimation of the hourly Solar Irradiance on a horizontal surface. Solar Energy 36, 132 (1986).

J. P. CHIOU and M. M. EI - NAGGAR, Optimun Slope for Solar Insolation on a fiat Surface Tilted toward the Ecuator in heating Season. Solar Energy 36, 471 (1986).

S. A. KLEIN, Calculation of monthly average insolation on tilted surface. Solar Energy 19, 325 - 329 (1977).

SAID M. A. IBRAHIM. Predicted and measured global solar radiation in Egypt. Solar Energy 35, 185 (1985).

N. A. VILLA NOVA, M.N. GOES RIBEIRO, CA. NOBRE, E. SA LATI. Radiacáo Solar em M anaus. A cta A mazónica 8 (3) 417 - 421 (1978)

J.C. OMETTO, Bioclimatología Vegetal, Editorial Agronómica Ceres Ltda. Sao Paulo (1981).

A. ANGSTROM, Solar and terrestrial radiation. Q.J.R. Met. SOC, SO. 121, 126 (1924).

J. GLOVER and J.S.F. MC CULLOCH. The empirical relation between solar radiation and hours of bright sunshine in the high altitud Tropics. Q.J.R. M et. Soc. 84 (359), 56 - 60 (1958).

L. OLDEMAN and FORERE A. study of the A groclimatology of the Humid Tropics of South East A sia W.M.O. Technical N ote N 0179. 
R.G. BARRY y R.S. CHORLEY. A tmósfera, Tiempo y Clima. Ediciones OM EGA S.A. (1972).

B. y H. LIU and R.C. JORDAN, The Interrelationship and characteristic distribución of direct, difusse and total solar radiation. Solar Energy 4, 1 (1960). 